\title{
IBBB Inter Frame Prediction of H.264 /AVC
}

\author{
Mohamed Al-Jammas \\ University of Nineveh
}

\begin{abstract}
As the world expanded around us and increased the popularity of the Internet by sending receiving, uploading or downloading the high definition videos, it was necessary to use a good technology to reduce the size of the dedicated video and specialized high-quality one. If the videos are send or receive, they need a wide bandwidth to capture this amount of information in the video. Based on the above, the H.264/AVC is a good technology that gives great results for encoding and decoding videos. This technology was developed jointly by (ITU-T) International Telecommunication Union-Telecommunication Standardization, and (ISO) International Organization for Standardization.
\end{abstract}

Our work involves applying the encoding and decoding process of the standard using MATLAB (2013Ra) program. The work is focusing in inter frame prediction using the (IBBB) frame pattern. The video that was subjected to encoding and decoding processing was (Xylophone video name) with (240X320) size and (30f/sec) as a bit rate.

\section{General Terms}

H.264/AVC,MATLAB.

\section{Keywords}

ITU-T, ISO,IBBB.

\section{INTRODUCTION}

Video coding is the process where the original video is convert to a format, which is compatible with many video player applications. At past decade, videos are stored in a magnetic tape or at an analogue format and with a development in science wheel, the (CD) Compact Disc was been invented then (DVD) Digital Video Disc was set. For the approval of this scientific development, the analogue format of the video was been replaced by the digital one. It becomes possible for all to watch and store the high-definition videos thanks to this scientific progress. The H.264/AVC is consider as an easy way to get encoding and decoding of any type of video because of its algorithm that adopts in encoding and decoding.

\section{THE H.264 ENCODER}

H.264 encoder is the new technological way to encode and reduce the size of the indicated video to be able to send it over the network or store it in storage media like DVD, blue ray, and USB flash memory. It contents many stages as shown in Figure (1).[1][2]

\author{
Rusul Nabeel \\ University of Mosul
}

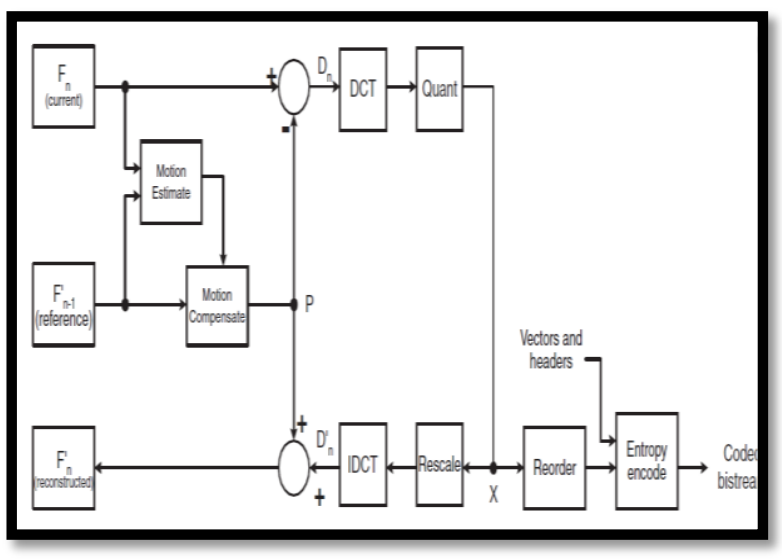

Fig.: 1 H.264 Encoder Path

The encoder has two paths (forward and reconstruction paths).

- In forward path, the input frame is process in units called macro blocks. Each macro block is encoded in an (intra or inter) mode and for each block in the macro block, a prediction is formed reconstructed picture samples. In the Inter mode, the prediction picture is formed by the motion compensated prediction from one or two previous reference picture(s), and the prediction reference for each macro block partition (in inter mode) may be chosen from a selection of past and future pictures (B frame types) that have already been encoded, reconstructed and filtered. The prediction picture is subtracted from the current block to produce a residual block that is transform (using a block transform) and quantized to a set of quantized transform coefficients, which are reorder and entropy encoded. The entropy encoded coefficients, together with side information required to decode each block within the macro block (prediction modes, quantize parameter, motion vector information, etc.) form the compressed bit stream which is passed to a Network Abstraction Layer (NAL) for transmission or storage [3][4].

- The reverse path in the encoder include decodes, (reconstructs) each block in a macro block to provide a reference frame for further predictions in the next. The coefficients are rescaled and inverse transformed to produce a difference block. The prediction block added to the previous decoded block to create a reconstructed block. The reconstructed reference picture is create from a series of blocks [5][6].

\section{THE H.264 DECODER}

The H.264 decoder is the reversible protocol of the encoder and it used to decode the encoding video come from the encoder. The decoder receives the compressed bit stream and decodes the data elements to produce a set of quantized 
coefficients, they are rescale and inverse transformed. Using the header information decoded from the bit stream, the decoder creates a prediction block. Figure (2) shows the H.264 decoder path [7][8].

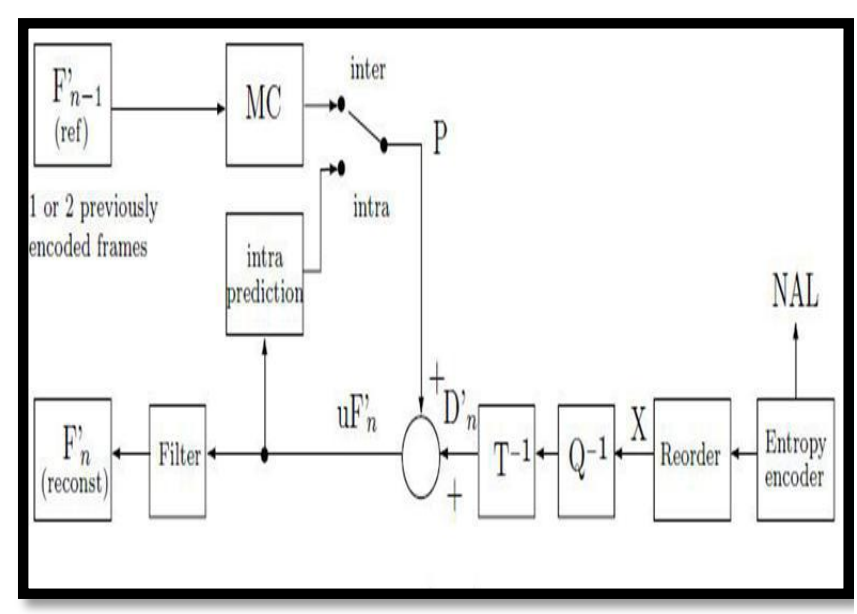

Fig: 2 H.264 Decoder Path

\section{THE MOTION ESTIMATION AND COMPONSATION}

In H.264/ AVC, each frame divided into of (16X16) pixels size which they called the macro blocks (MB). Each macro block is encoded using blocks of pixels encoded within the current frame or (Intra frame) coding or by using blocks of pixels in previous or future encoded frames (Inter frame) coding like (P and $\mathrm{B}$ frames) [9][10].The process of finding the best matching of pixels block in the inter frame is called the Motion Estimation (ME) and the displacement vector between two blocks is the Motion Vectors (MV) of the block. The motion estimation is computationally expensive process when the search was doing at every pixel position over different reference frames. There are several different integer search algorithm used to find the best matching like (Full search algorithm, 2D-log algorithm, three steps algorithm and etc.)

\subsection{The 2D-Log Block Matching Algorithm}

This algorithm has steps to find best matching. The first step includes (select an initial step size(s) and calculate the error for the block at the center of the search area and four points at (x) and (y) axis at distance (s) from center). The second step, if the position of the best match at the center of the candidate block, keep the center unchanged and reduce the step size by half, otherwise the best match becomes the center so repeat the first step. In the last step, if the step size value becomes (1), then all the (8) neighbor blocks around the collected center will be checked to find the best match. The 2D-log search algorithm has lesser search points than TSS, yet its prediction is more accurate, it defines the step size at the beginning and terminates if the step size is equal to one. The $2 \mathrm{D}-\log$ has complexity is about $((\log (\mathrm{p} / 2))$ where $\mathrm{p}$ is the search area size [20]. Figure (3) shows the 2D-Log algorithm [11].

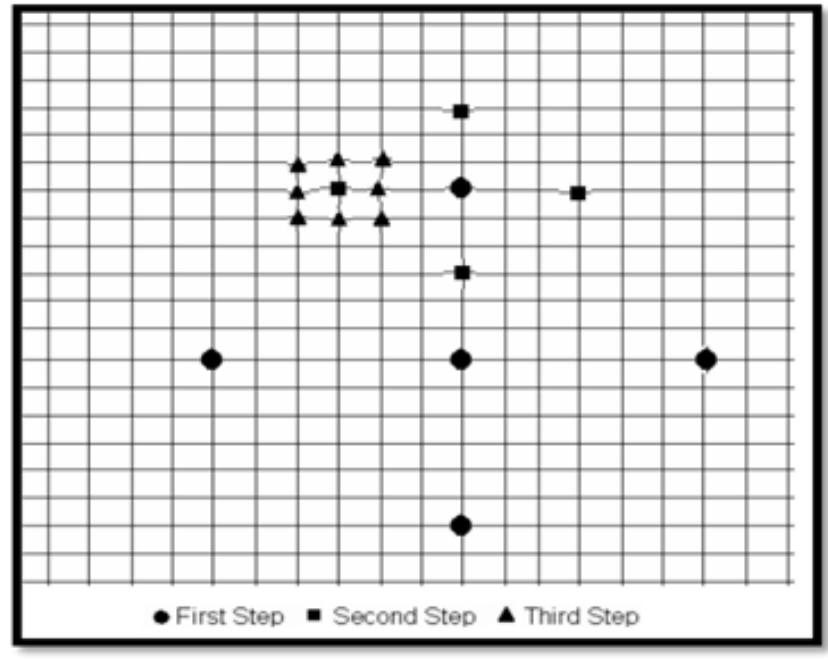

Fig: 3 2D-Log Algorithm

\subsection{Motion Vector}

MV defined as the displacement between the current block and the best matching block in the search area in the reference frame. It is the successive key of motion estimation and it is a directional pair representing the displacement in the horizontal direction (x-axis) and vertical direction (y-axis). The maximum value of MV determined by the search range. Traditionally one MV produced from each macro block in the frame.

\section{IBBB INTER FRAME IN MATLAB}

In order to achieve good results in compression and decompression, it was necessary to design the H.264 encoder and decoder. Our work is focusing on implement the H.264/AVC encoder and decoder in MATLAB, which is the best program available to simulate the reality. This H.264 encoder and decoder model has very good specifications with (IBBB) patterns of frame.

\subsection{H.264 Encoder In Matlab}

In order to begin coding process through the encoder there are several stages to follow. The original video is in (RGB) color space and it is good idea to convert it into (YUV) color space because the YUV contracts the size of the frame. It depends on the idea that the human eye is affect by lighting more than gradient change colors. The colors components (Chroma) and lighting components (Luma) are sampled based on weight that specific by (4:2:0) sample format. In real world, videos are in RGB where each colors (Red, Green and Blue), are sample equally in same resolution. In new space of color, the luminance is separated from the colors, and representing luminance with a higher resolution than colors. The video is initialize and it is ready to be encode by several steps. The video frame is predicted in an inter prediction.[12]

\subsection{Inter Frame Prediction (IBBB)}

The Inter frame prediction aimed is to remove the temporal redundancy of the video. The work is aiming to find a good match for the current block from the previously coded image, and the block from the future and previous coded image, this is the work of the (IBBB) frame pattern. The inter prediction tools contribute to the improved the compression efficiency of the H.264/AVC standard. The inter prediction includes steps that are representing the power of the H.264/ AVC because they have the key of compression. They exploit the high redundancy that happened between successive frames in the 
video. To achieve this goal, a block matching algorithms are used. The real world objects can move, jumping, rotate, etc. These movements cannot be observe directly, but the light reflected from the object surfaces and projected onto an image. There are some noises happened when the image is taken by a video camera. Motion compensation is the technique that uses the redundancy between frames in a video sequence to compress the data. Once the motion estimation has done, the algorithm of the block matching only transmits the difference between the successive frames by applying the motion compensation. Figure (4) shows the frame of the video after 2D-log block matching algorithm including (motion estimation and compensation).

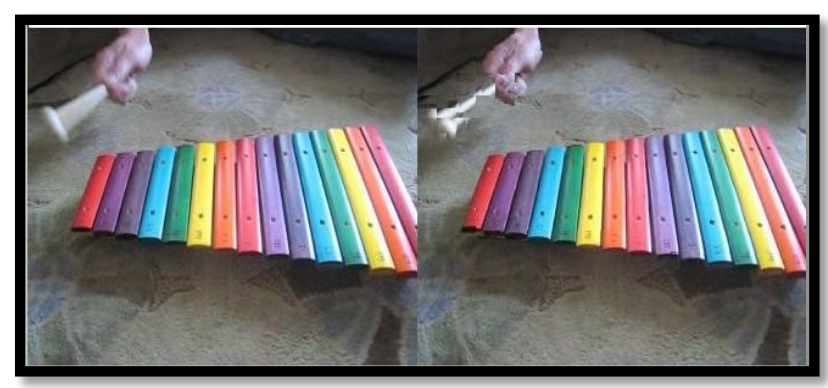

Fig: 4 Video Frame after 2D-Log Algorithm

\subsection{Motion Vector of Frames}

Each frame after motion estimation and compensation turns into groups of coefficients and this can be explained as motion vector. All these coefficients and motion vectors that in turn will be performed on them transformation and quantization processing. Figure $(5, a, b)$ shows the frames and its motion vector.

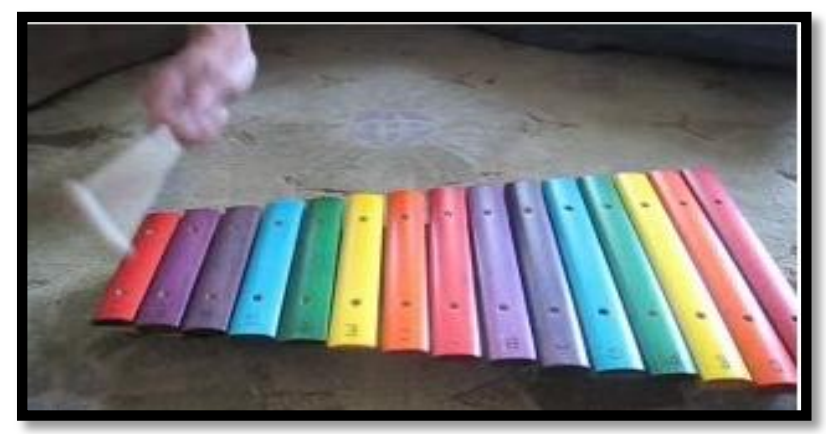

Fig.: 5a Frame \#2

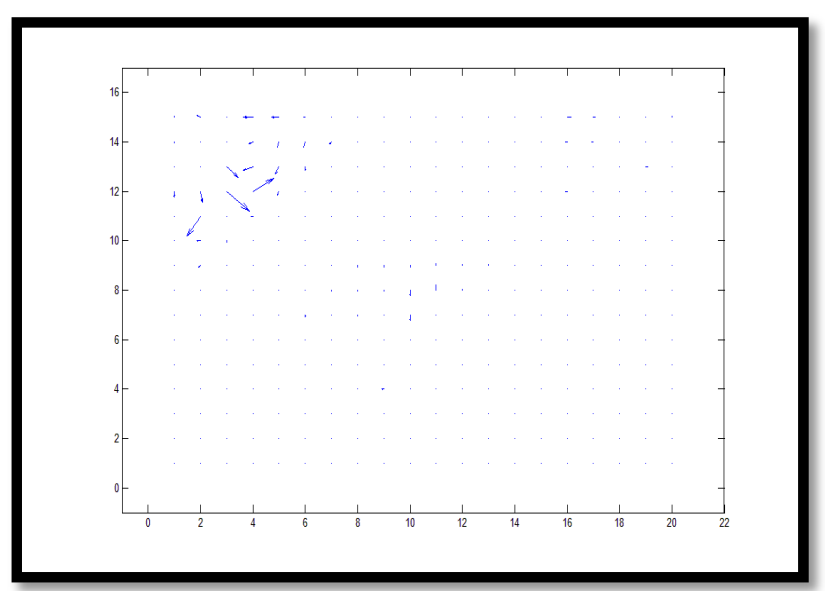

Fig.: 5b Motion Vector Frame \#2

\subsection{Transformation and Quantization}

In the encoder, after applying the motion estimation and compensation in the data and gain residual data frame, transformation and quantization units receive the residual data frame and process them. The transform unit reduces the temporal redundancy. The data after transformation is easy to compress rather than untransformed one. The results are called transform coefficients and they quantized by the quantization unit. There are many types to achieve the transformation like (2D-DCT).

\subsection{H.264 Decoder in Matlab}

The H.264 decoder receives the compressed video and decode it to gain the original video. The decoder receives the quantized data, it rescale them by multiplying with quantization parameter $\mathrm{QP}$, doing inverse transform, reconstruct it and convert the result to the RGB color space then the video is return. Figure $(6 . a, b)$ shows the video frames after decoding it and comparing it with original one.

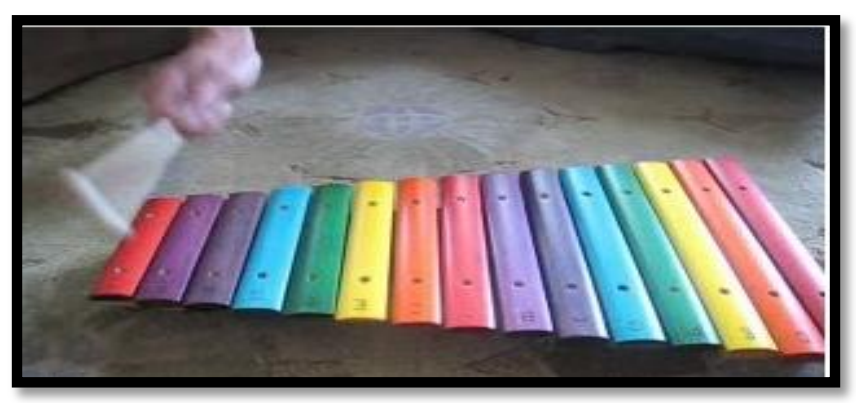

Fig.: 6a Original frame \#2

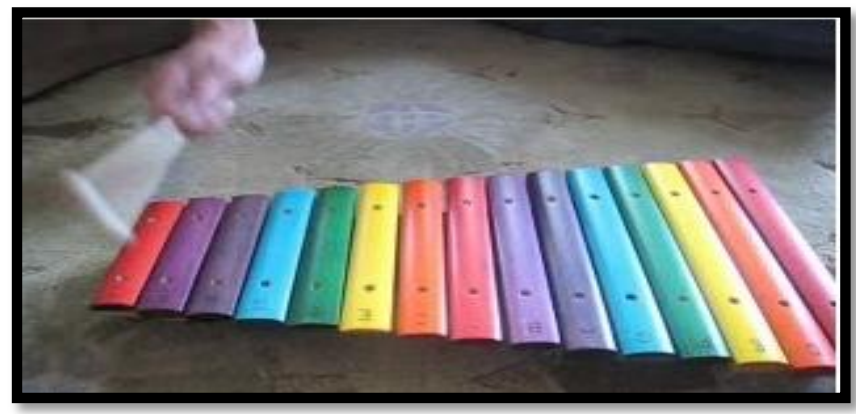

Fig.:6b Decoding Frame \#2

\section{CONCLUSIONS}

Through our design of the H.264 encoder and the decoder, we found that there is a decoder inside the encoder to get the reference frames for next compression frame. The Compression rate in (IBBB) is $71 \%$, this is a good result we gain and better than in (IPPP) which is equal to $50 \%$. The time it takes to complete the overall encoding process is about $0.2142 \mathrm{sec}$ and the time it takes to complete the overall decoding process is about $0.0994 \mathrm{sec}$.

\section{REFERENCES}

[1] Darshankumar Shah, B.E, H.264 MOTION ESTIMATION AND MOTION COMPENSATION, Thesis of Master of Science in Electrical and Electronic Engineering at California State University, Sacramento, 2011.

[2] By STEPHEN, A Fine Grained Many-Core H.264 Video Encoder, Thesis of Master of Science in Electrical and Computer Engineering in the University of California, 
THE UY LE B.S., March, 2007.

[3] YIM, Ka Yee, Video Decoder for H.264/AVC Main Profile, Power Efficient Hardware Design, A Thesis of Master of Philosophy in Electronic Engineering, Chinese University of Hong Kong, August 2011.

[4] Samia Sharmin Shimu, Performance Analysis of H.264 Encoder for High-definition Video Transmission over Ultra-Wideband Communication Link, Thesis of Master of Science in the Department of Electrical and Computer Engineering ,University of Saskatchewan Saskatoon, May 2010.

[5] Kermin Fleming, Chun-Chieh Lin, Jamey Hicks , H.264 Decoder: A Case Study in Multiple Design Points, 6th ACM/IEEE International Conference on Formal Methods and Models for Co-Design PP.165-174, 20JUNE 2008.

[6] Michael , Anthony Joch, Faouzi Kossentini and Antti Hallapuro, H.264/AVC Baseline Profile Decoder Complexity Analysis, IEEE Transactions on Circuits and Systems for Video Technology, VOL. 13, NO. 7, PP 704-716, JULY 2003.

[7] A. Ben Atitallah, H. Loukil ,Nouri, FPGA Design for H.264/AVC Encoder, International Journal of Computer Science, Engineering and Applications (IJCSEA) Vol.1, No.5, PP 119-138, October 2011.
[8] Jignesh Patel, Haresh Suthar, Jagrut Gadit, Parul ,VHDL Implementation of H.264 Video Coding Standard , International Journal of Reconfigurable and Embedded Systems (IJRES),Vol. 1, No. 3, pp. 95-102, November 2012.

[9] Gary J. Sullivan, Pankaj Topiwala, and Ajay Luthra, The H.264/AVC Advanced Video Coding Standard, Overview and Introduction to the Fidelity Range Extensions, SPIE Conference on Applications of Digital Image Processing XXVII Special Session on Advances in the New Emerging Standard H.264/AVC, August 2004.

[10] LI Man Ho, Variable Block Size Motion Estimation Hardware for Video Encoders, Thesis of Master of Philosophy in Computer Science and Engineering, The Chinese University of Hong Kong, November 2006.

[11] Iain E. Richardson, The H.264 Advanced Video Compression Standard, John Wiley \& Sons, Ltd., Second Edition, Vcodex Limited, UK, 2010.

[12] Iain E. G. Richardson, H.264 and MPEG-4 Video Compression Video Coding for Next-generation Multimedia, John Wiley \& Sons Ltd, First Edition, The Atrium, Southern Gate, Chichester, West Sussex PO19 8SQ, England ,2003. 